$\begin{array}{ll}\alpha_{0} & =\text { initial amplitude of disturbance } \\ \mu & =\text { viscosity of liquid } \\ \rho & =\text { density of liquid } \\ \sigma & =\text { surface tension } \\ \omega & =\text { angular velocity of disk }\end{array}$

[cm]

$[\mathrm{g} / \mathrm{cm} \cdot \mathrm{sec}]$

$\left[\mathrm{g} / \mathrm{cm}^{3}\right]$

[dyn $/ \mathrm{cm}]$

$\left[\mathrm{sec}^{-1}\right]$

\section{Literature Cited}

1) Kamiya, T.: J. Chem. Eng. Japan, 5, 391 (1972).

2) Kurabayashi, T.: Trans. Japan. Soc. Mech. Engrs., 25, 1252, 1259, 1266 (1959); 26, 1536 (1960).

3) Weber, C.: Angew. Math. Mech., 11, 136 (1931).

\title{
GROUP THEORETIC APPROACH TO SIMILARITY SOLUTIONS OF CHEMICAL BOUNDARY LAYER
}

\author{
K. M. CHAKRABARTI* AND S. K. GHOSHAL ${ }^{1}$ \\ Department of Mathematics, Regional Engineering College, Durgapur-9, West Bengal, India
}

In this paper group theoretic approach to similarity solution of chemical boundary layer has been employed. We have seen that if $n=1$ then in general a similarity solution is possible when the flow is accompanied by $n$ th-order homogeneous volume reaction.

Chemical boundary layer may be described by the following equations ${ }^{1)}$ :

$$
\begin{aligned}
\phi_{1} & \equiv \frac{\partial u}{\partial t}+u \frac{\partial u}{\partial x}+v \frac{\partial u}{\partial y}-\frac{\partial^{2} u}{\partial y^{2}}=0 \\
\phi_{2} & \equiv \frac{\partial u}{\partial x}+\frac{\partial v}{\partial y}=0 \\
\phi_{3} & \equiv \frac{\partial c}{\partial t}+u \frac{\partial c}{\partial x}+v \frac{\partial c}{\partial y}-\frac{1}{S c} \frac{\partial^{2} c}{\partial y^{2}}-\frac{L^{2} K}{\nu} c^{n}=0
\end{aligned}
$$

Where $S c=$ Schmidt's number, $\nu=$ kinematic viscosity $c=$ concentration of the solute, $L=$ characteristic length $k=$ a suitable constant.

It can easily be verified that the expressions $\phi_{1}, \phi_{2}, \phi_{3}$ remain constant conformally invariant under the group of transformation ${ }^{2,3)}$

$$
\left.\begin{array}{l}
\bar{x}=a^{\alpha_{1}} n, \quad \bar{y}=a^{\alpha_{2}} y, \quad \bar{t}=a^{2 \alpha_{2}} t \\
\bar{u}=a^{\alpha_{1}-2 \alpha_{2}} u, \quad \bar{v}=a^{-\alpha_{2}} v, \quad \bar{c}=a^{2 \alpha_{2} /(1-n)} c
\end{array}\right\}
$$

Thus we see that $n \neq 1$.

Let us choose $\eta_{1}$ and $\eta_{2}$ as follows:

where $p=\alpha_{1} / \alpha_{2}$.

$$
\eta_{1}=n^{-p} y, \quad \eta_{2}=x^{-2 p} t
$$

It can easily be verified that $\eta_{1}$ and $\eta_{2}$ are absolute invariants under the group of transformations (4).

The dependent variables can similarly be transformed as follows:

$$
\left.\begin{array}{l}
u=x^{1-2 p} F_{1}\left(\eta_{1}, \eta_{2}\right) \\
v=x^{-p} F_{2}\left(\eta_{1}, \eta_{2}\right) \\
c=x^{2 p /(1-n)} F_{3}\left(\eta_{1}, \eta_{2}\right)
\end{array}\right\}
$$

Received April 27, 1974

1 Jadavpur Univ., Calcutta, India
With the help of relations (5) and (6) Eqs. (1), (2) and (3) respectively become

$$
\begin{gathered}
\frac{\partial F_{1}}{\partial \eta_{2}}+F_{1}\left\{(1-2 p) F_{1}-p\left(\eta_{1} \frac{\partial F_{1}}{\partial \eta_{1}}+2 \eta_{2} \frac{\partial F_{1}}{\partial \eta_{2}}\right)\right\} \\
+F_{2} \frac{\partial F_{1}}{\partial \eta_{1}}-\frac{\partial^{2} F_{1}}{\partial \eta_{1}^{2}}=0 \\
(1-2 p) F_{1}-p\left\{\eta_{1} \frac{\partial F_{1}}{\partial \eta_{1}}+2 \eta_{2} \frac{\partial F_{1}}{\partial \eta_{2}}\right\}+\frac{\partial F_{2}}{\partial \eta_{1}}=0 \\
\frac{\partial F_{3}}{\partial \eta_{2}}+F_{1}\left\{\frac{2 p}{(1-n)} F_{3}-p\left(\eta_{1} \frac{\partial F_{3}}{\partial \eta_{1}}+2 \eta_{2} \frac{\partial F_{3}}{\partial \eta_{2}}\right)\right\} \\
+F_{2} \frac{\partial F_{3}}{\partial \eta_{1}}-\frac{1}{S c} \frac{\partial^{2} F_{3}}{\partial \eta_{1}^{2}}-\frac{L^{2} K}{\nu} F_{3}^{n}=0
\end{gathered}
$$

Using the independent variables $\eta_{1}, \eta_{2}$ and the dependent variables $F_{1}, F_{2}, F_{3}$ we set up a new oneparameter transformations group as follows:

$$
\begin{aligned}
& \bar{\eta}_{1}=b^{\varepsilon_{1}} \eta_{1}, \quad \bar{\eta}_{2}=b^{2 \varepsilon_{1}} \eta_{2}, \quad \bar{F}_{1}=b^{-2 \varepsilon_{1}} F_{1} \\
& \bar{F}_{2}=b^{-\varepsilon_{1}} F_{2}, \quad \bar{F}_{3}=b^{2 \varepsilon_{1} /(1-n)} F_{3}
\end{aligned}
$$

It can easily be verified that the left-hand sides of Eqs. (7), (8) and (9) remain constant conformally invariant under the transformations (10). It can be verified that transformation of the independent variables given by

$$
\eta=\eta_{1} \eta_{2}^{-1 / 2}
$$

is an absolute invariant under the group of transformations (10).

The transformations involving the dependent variables are as follows:

$$
F_{1}=\eta_{2}^{-1} H_{1}(\eta), \quad F_{2}=\eta_{2}^{-1 / 2} H_{2}(\eta), \quad F_{3}=\eta_{2}^{1 /(1-n)} H_{3}(\eta)
$$

Employing the transformation (12) and the relation (11) in Eqs. (7), (8), (9) we get respectively:

$$
\begin{aligned}
& \left\{H_{1}+(1 / 2) \eta H_{1}^{\prime}\right\}-H_{1}^{2}-H_{2} H_{1}^{\prime}+H_{1}^{\prime \prime}=0 \\
& H_{1}+H_{2}^{\prime}=0
\end{aligned}
$$




$$
\begin{aligned}
& {\left[\frac{1}{(1-n)} H_{3}-\frac{1}{2} \eta H_{3}^{\prime}\right]+H_{2} H_{3}^{\prime}-\frac{1}{S c} H_{1}^{\prime \prime}} \\
& -\frac{L^{2} K}{\nu} H_{3}^{n}=0
\end{aligned}
$$

Thus we see that the chemical boundary layer Eqs. (1), (2) and (3) are reduced respectively to Eqs. (13), (14) and (15), which are ordinary differential equations. Hence similarity solution of chemical boundary layer equations is possible by group theoretic approach. We have already shown that the above results are valied when $n \neq 1$.
The boundary conditions for $H_{1}, H_{2}, H_{3}$ can be fixed at this stage and the Eqs. (13)-(15) can be solved for $H_{1}, H_{2}, H_{3}$ with the help of the boundary conditions.

\section{Literature Cited}

1) Bird, Lightfoot and Stewart: "Transport Phenomena", John Wiley \& Sons, Inc.

2) Morgan, A. J. A.: Discussion of "Possible Similarity Solution of the Laminar, Incompressible, Boundary-Layer Equations", Trans. A.S.M.E., 80, 7, (1958).

3) Birkhoff, G.: "Hydrodynamics", Dover Publications, Inc., New York (1955)

\title{
MINIMUM WETTING RATE ON WETTED-WALL COLUMN - IN THE ABSENCE OF MASS AND HEAT TRANSFER-
}

\author{
KAZUNORI WATANABE*, TsuYoshi MUNAKATA AND AKIRA MATSUDA \\ Department of Chemical Engineering, Kyushu University, Fukuoka, 812
}

\section{Introduction}

In falling-film mass or heat transfer units, it is important to make a stable liquid flow over the wall to ensure the wetted area contributing to the cransfer process. To date, regarding wetted-wall cal packed columns with or without mass and/or heat transfer, several workers have measured the minimum wetting rates (M.W.R.) of two cases, i.e. one where the flow rate is reduced until the uniform film just starts to rupture (Case I), and another where the rate is increased until initially dry surface is wetted completely (Case II). Also, a few workers have theoretically and experimentally presented equations to predict the M.W.R. These equations are shown in

Received May 17, 1974.

A part of this paper was presented at the 7th Autumn Meeting of The Soc. of Chem. Engrs., Japan, at Nagoya, Oct., 1973.
Table 1. Nevertheless, even for the isothermal condition there is a great discrepancy in the measured values, and theoretical treatments of the film rupture have not necessarily been successful. The present work attempts to give satisfactory reasons for such inconsistency.

\section{Experimental Apparatus and Procedure}

Experiments have been done for water, $n$-propanolwater mixture $(8.62 \mathrm{~mole} \%)$ and ethyl oxalate in the two cases mentioned above by use of $3.5 \mathrm{~cm}-O . D$. smooth glass tubes. The apparatus is shown in Figs. 1a and 1b. Six specimen tubes have been prepared, each of which has a different top shape, as shown in Fig. 2. The tube was cleaned with chromic acid mixture, and then washed with distilled water before the start of an experiment. This pretreatments was given to all tubes.

A liquid from the head tank was supplied to the

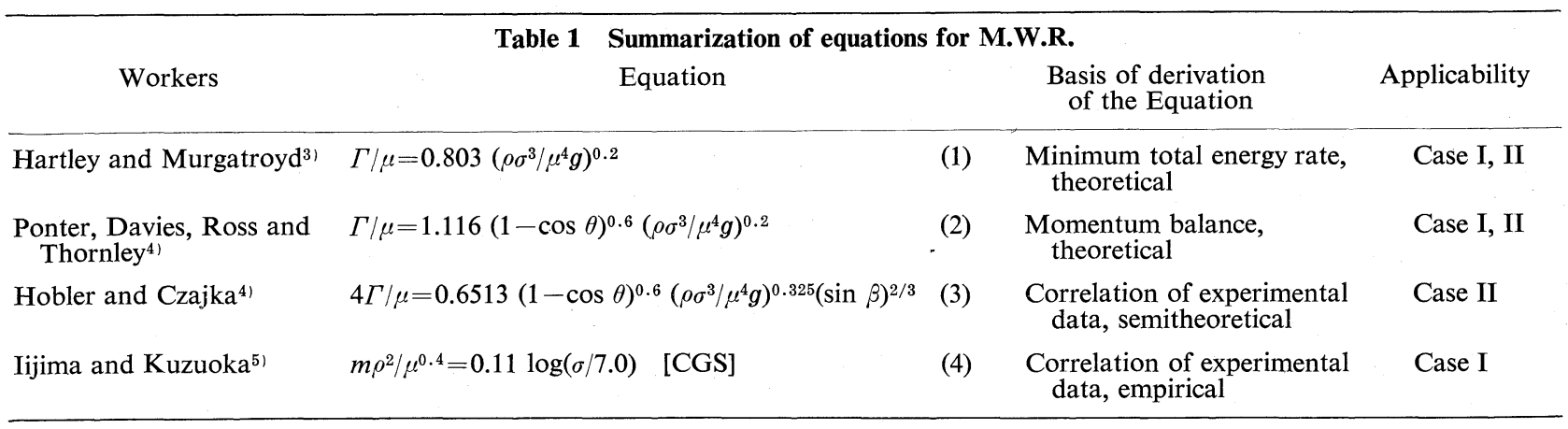

